\title{
Selection for Increased Penicillin Titre Following Hybridization of Divergent Lines of Aspergillus nidulans
}

\author{
By I. N. SIMPSON† AND C. E. CATEN* \\ Department of Genetics, University of Birmingham, Birmingham B15 2TT, U.K.
}

(Received 11 March 1981)

\begin{abstract}
Sexual hybridization of two divergent lines of Aspergillus nidulans, which had been selected for increased penicillin titre through successive cycles of mutagenesis, released considerable variation for this character. The recovery of segregants with titres equivalent to that of the unselected ancestor suggested that mutations in different genes had been selected in the two lines. However, complementary segregants with substantially improved titres were not found, indicating interactions, probably of a duplicate nature, among the induced mutations. All the genetic variation released by hybridization was fixed following two generations of selection for high titre, but only a small gain over the initial selection lines was achieved. Hybridization of divergent strains has been widely advocated as a means of strain development. The failure to achieve the anticipated gains in this programme is attributed primarily to the unfavourable interactions amongst the induced mutations. Whether similar interactions occur generally in crosses between strains selected by mutagenesis remains to be established and will be an important factor in determining the contribution of recombinational approaches to yield improvement.
\end{abstract}

\section{IN TRODUCTION}

There has been considerable discussion of the use of recombination for strain improvement in antibiotic-producing micro-organisms (for example, Calam, 1964; Sermonti, 1969; Alikhanian, 1970; Elander \& Espenshade, 1976), and systems of recombination have been developed in several industrially important species (for review, see Hopwood \& Merrick, 1977). Particularly attractive is the possibility of combining yield-enhancing alleles from divergent sources into one strain through hybridization followed by selection amongst the progeny. However, extensive attempts to breed improved strains of Penicillium chrysogenum through parasexual hybridization were largely unsuccessful (Sermonti, 1959; Macdonald et al., 1965; Macdonald, 1966; Elander et al., 1973) and it is generally considered that recombination has to date contributed little to practical strain improvement (Elander et al., 1977). Possible reasons for the failure of these initial attempts at breeding have since been identified (Ball, 1971, 1973), and currently there is a renewed interest in recombination as a means of strain improvement (Hopwood, 1977; Hopwood \& Chater, 1980; Ball, 1978). The practical value of hybridization has been recently demonstrated by the isolation from a cross between two divergent lines of Cephalosporium acremonium of a segregant showing a $40 \%$ increase in cephalosporin C titre, together with other improvements (Hamlyn \& Ball, 1979).

Soller (1980) developed models for the response of microbial populations to selection programmes involving mutagenesis alone or mutagenesis in combination with hybridization. Comparisons of the response to selection predicted by these two models indicated that under certain conditions the gains in the combined programme may exceed those in the mutagenesis

† Present address: Microbiology Division, Glaxo Group Research Ltd, Greenford, Middlesex UB6 0HE, U.K. 
programme and therefore that it may pay to divide the mutation stages into two divergent lines which can subsequently be crossed. The extent to which the conditions favouring hybridization will be realized in practice can only be determined by experiment. This paper describes hybridization and selection experiments which are very similar to the model programmes considered by Soller (1980). The results provide tests for many of the critical conditions of the models and permit some evaluation of the benefits of recombination in yield-improvement programmes.

Two divergent strains of Aspergillus nidulans, A6-9 and B6-27, were independently selected from isogenic ancestral strains by recurrent mutagenesis (Simpson \& Caten, 1979b). Selection was for increased penicillin titre in shake-flask fermentations and each strain showed around a threefold increase in productivity over its unselected ancestor. Although $A$. nidulans is not a commercial antibiotic producer it has proved valuable as a model organism for genetic studies of penicillin production (Macdonald \& Holt, 1976), and the derivation of A6-9 and B6-27 was closely comparable to the procedure used industrially for yield improvement in antibiotic-producing organisms (Davies, 1964; Demain, 1973; Elander \& Espenshade, 1976). Information obtained from studies of A6-9 and B6-27 should therefore be directly relevant to the genetics of antibiotic yield in improved industrial strains. Backcrosses of the two selected strains to the ancestral genotype revealed that the increased yield of each was due to a polygenic system involving both additive and non-additive gene action (Simpson \& Caten, 1980). A diploid constructed between A6-9 and B6-27 gave a low titre implying that the titre-increasing mutations (' + ' mutations) in the two lines were non-allelic (Simpson $\&$ Caten, 1980). We here report an attempt to select recombinants with further increased penicillin titre from a cross between A6-9 and B6-27. Following the initial cross, two generations of selection and hybridization were initiated from the progeny population. Sexual rather than parasexual hybridization was used because (1) it avoids the need to select haploid segregants and therefore facilitates taking a random sample of progeny (Caten \& Jinks, 1976), (2) the theory and methods of quantitative genetics can be readily applied (Caten, 1979 ) and (3) the rate of intrachromosomal recombination is much higher.

\section{METHODS}

Strains. The origins of the ancestral strains of Aspergillus nidulans, strain A (ATCC 42369) and strain B (ATCC 42370), have been described by Simpson \& Caten (1979a) who referred to them as $7 / 142$ and $7 / 151$, respectively. Strain A carries the marker biA1 and strain B carries the markers pabaA1; $w A 3$. These markers do not significantly affect penicillin titre under the fermentation conditions used (Simpson \& Caten, 1980). Strains A and B are both descended from the 'Glasgow' wild-type, NRRL 194. Both carry the standard chromosome complement and are isogenic for genes affecting penicillin titre (Simpson \& Caten, 1980). The improved strains

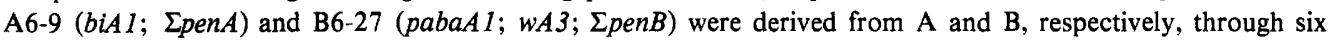
cycles of mutation and selection for increased penicillin titre (Simpson \& Caten, 1979 b). Averaged over at least six separate fermentations these strains gave the following mean penicillin titres (range in parentheses): $\mathrm{A}=$ $5.7 \mathrm{u} \mathrm{ml}^{-1}(3.0-7.4) ; \mathrm{A} 6-9=14.2 \mathrm{u} \mathrm{m}^{-1}(11.2-20.5) ; \mathrm{B}=5.9 \mathrm{u} \mathrm{ml}^{-1}(3.2-7.8) ; \mathrm{B} 6.27=15.5 \mathrm{u} \mathrm{ml}^{-1}$ (11.0-19-6). $\Sigma$ penA and $\Sigma$ penB denote the '+' mutations accumulated in A6-9 and B6-27, respectively. Other gene symbols are standard for $A$. nidulans (Clutterbuck, 1973).

General. Media, methods of culture, fermentation and bioassay procedures and statistical analyses were all as described previously (Simpson \& Caten, 1979a,b). Crosses were carried out by the sexual cycle using single perithecium analysis as described by Merrick \& Caten (1975).

\section{RES ULTS}

\section{Hybridization of $A 6-9$ and $B 6-27$}

Strains A6-9 and B6-27 were crossed twice and a sample of progeny taken on each occasion. The first sample, designated S0, formed the base population for a subsequent programme of hybridization and selection and contained 95 randomly selected progeny. The second sample, designated SX, was chosen to test for any association between the induced 


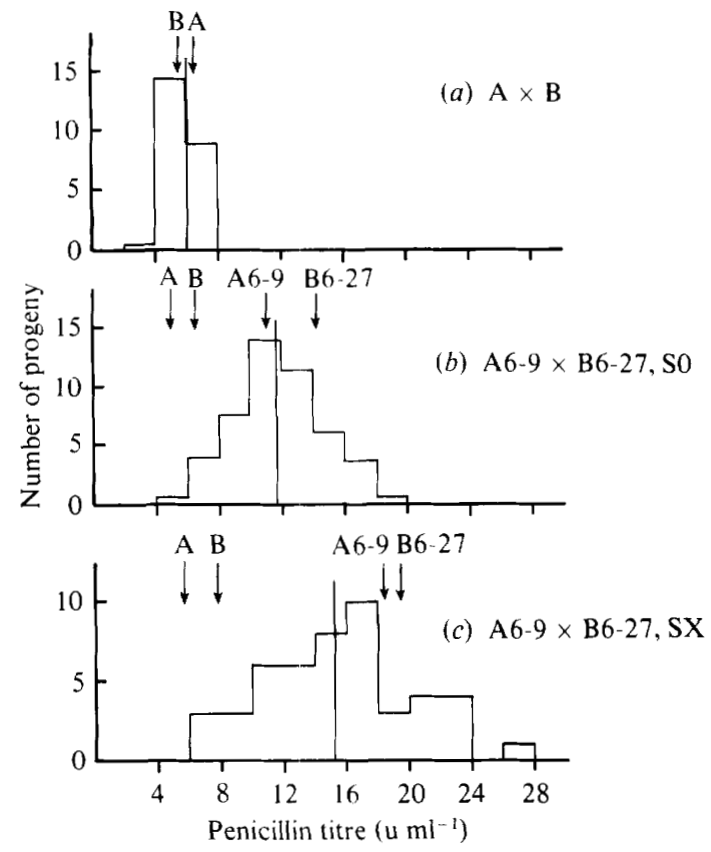

Fig. 1. Distribution of penicillin titres among progeny from crosses $\mathrm{A} \times \mathrm{B}(a), \mathrm{A} 6-9 \times \mathrm{B} 6-27, \mathrm{~S} 0(b)$ and A6-9 $\times$ B6-27, SX (c). The titres of A, B, A6-9 and B6-27 are shown by arrows. The bars indicate the progeny mean titres.

titre mutations and the markers (biA, pabaA, wA) and consisted of six progeny of each of the eight marker genotypes arising from the cross. The titres of the S0 progeny were determined in two separate fermentation experiments. In both experiments the 95 progeny were each fermented in one flask. These flasks were sampled and assayed following the procedure previously used for populations of around 100 strains (Simpson \& Caten, 1979 b). The SX progeny were each fermented in two replicate flasks in a single experiment and were assayed following the general procedure for populations of around 50 strains (Simpson \& Caten, $1979 a$ ). Two replicate flasks of A, B, A6-9 and B6-27 were included in all three experiments.

The distributions of the progeny titres in samples SO (mean of the two experiments) and SX are shown in Fig. $1(b)$ and $(c)$, respectively. Results previously obtained for a sample of progeny from the cross between the ancestral strains (Simpson \& Caten, 1980) are included as a control (Fig. 1a). Strains A and B performed similarly in all experiments but A6-9 and B6-27 gave higher titres in the SX fermentation than in the two S0 fermentations. In the absence of a consistent effect upon the performance of these four standard strains no attempt was made to correct the progeny titres for differences in the fermentation conditions between experiments. All three progeny populations showed continuous and unimodal distributions (Fig. 1). The means of the two A6-9 $\times$ B6-27 progenies were higher than that for the control population, confirming the genetic basis of the high titres of the selected strains. Furthermore, the range of titres amongst the progeny of A6-9 $\times$ B6-27 was markedly greater than that amongst the control population, reflecting the segregation and recombination of alleles affecting penicillin production. In both A6-9 $\times$ B6-27 crosses a few progeny were recovered with titres in excess of both parental isolates; however, the majority were less productive than their parents and a few produced no more penicillin than the unselected ancestors $\mathrm{A}$ and $\mathrm{B}$ (Fig. $1 b, c)$.

Analyses of variance for the data from the three progeny samples are summarized in Table 1. The variation within each progeny sample was partitioned into that between and that 
Table 1. Summary of analyses of variation in penicillin titre $\left(\log u \mathrm{ml}^{-1}\right)$ among progenies from $A \times B$ and $A 6-9 \times B 6-27$

(a) Tests of significance

\begin{tabular}{|c|c|c|c|c|c|c|c|c|c|c|}
\hline \multirow[b]{2}{*}{ Cross } & \multicolumn{2}{|c|}{ Between progeny } & \multicolumn{2}{|c|}{$\begin{array}{l}\text { Between marker } \\
\text { genotypes }\end{array}$} & \multicolumn{2}{|c|}{$\begin{array}{l}\text { Within marker } \\
\text { genotypes }\end{array}$} & \multicolumn{2}{|c|}{ Between blocks } & \multicolumn{2}{|c|}{ Progeny $\times$ blocks } \\
\hline & d.f. & M.S. & d.f. & M.S. & d.f. & M.S. & d.f. & M.S. & d.f. & M.S. \\
\hline $\mathbf{A} \times \mathbf{B} \dagger$ & 47 & 0.010 & 7 & 0.016 & 40 & 0.009 & 1 & $0 \cdot 149^{*}$ & 47 & 0.012 \\
\hline $\mathrm{A} 6-9 \times \mathrm{B} 6-27, \mathrm{~S} 0$ & 94 & $0.044^{*}$ & 7 & 0.065 & 87 & $0.043^{*}$ & 1 & $0.691^{*}$ & 94 & 0.017 \\
\hline A6-9 $\times$ B6-27, SX & 47 & $0.042 *$ & 7 & 0.031 & 40 & $0.044^{*}$ & 1 & 0.001 & 47 & 0.012 \\
\hline
\end{tabular}

(b) Components of variance

$\begin{array}{cccc}\text { Cross } & \text { Genetic }\left(\sigma_{G}^{2}\right) & \text { Blocks }\left(\sigma_{B}^{2}\right) & \text { Environmental }\left(\sigma_{E}^{2}\right) \\ \mathrm{A} \times \mathrm{B} \dagger & 0.000 & 0.003 \ddagger & 0.012 \\ \mathrm{~A} 6-9 \times \mathrm{B} 6-27, \mathrm{~S} 0 & 0.014 \ddagger & 0.007 \ddagger & 0.017 \\ \mathrm{~A} 6-9 \times \mathrm{B} 6-27, \mathrm{SX} & 0.015 \ddagger & 0.000 & 0.012 \\ \text { d.f., degrees of freedom; M.S., mean square. } & * P<0.001 . \\ \dagger \text { Data from Simpson \& Caten (1980). } & \$ \text { Significantly different from zero. }\end{array}$

within marker genotypes. In no case was the between marker genotypes mean square (M.S.) significantly greater than the within marker genotypes M.S. This lack of any effect of the three markers on penicillin titre is consistent with the backcrosses of A6-9 and B6-27 (Simpson \& Caten, 1980) and means that the between and within marker genotype M.S. values can be pooled to give a between progeny M.S. This between progeny M.S. was highly significant for the two progenies from A6-9 $\times \mathrm{B} 6-27$ but not for $\mathrm{A} \times \mathrm{B}$ (Table $1 a$ ). S0 and SX gave similar estimates for the amount of genetic variation $\left(\sigma_{G}^{2}\right)$ released in A6-9 $\times$ B6-27 which clearly contrast with the zero estimate for $\mathrm{A} \times \mathrm{B}$ (Table $1 b$ ). The higher estimates of the environmental component $\left(\sigma_{E}^{2}\right)$ and of the variance between blocks $\left(\sigma_{B}^{2}\right)$ for S0 (Table $1 b$ ) may be attributed to the fact that the replicates in this case were fermented in different experiments. These analyses confirm the segregation of alleles affecting penicillin titre in the cross between the divergent selection lines. Since the cross between the ancestral strains does not segregate, these genetic differences between A6-9 and B6-27 must have been induced during the mutagenesis programmes.

The progeny of A6-9 $\times$ B6-27 were not symmetrically distributed about the parental mean as expected with solely additive gene action (Caten \& Jinks, 1976). In both samples there was an excess of progeny with titres below the mid-parental value and this was particularly marked for SX (Fig. 1b,c). The deviation of the progeny mean from the mid-parental value gives an estimate of the contribution of directional non-additive gene effects $[i]$ to the parental phenotypes (Mather \& Jinks, 1971; Caten, 1979). Whether these estimates are significantly different from zero can be tested with a ' $t$ ' test. Non-additive gene effects (epistasis) were not apparent in $\mathrm{A} \times \mathrm{B}$ (Table $2 a$ ). (This was expected since the overall analysis indicated no genetic variation in this cross.) In both samples of progeny from A6-9 $\times$ B6-27, however, the progeny mean was less than the mid-parental value, giving positive estimates of $[i]$ (Table $2 a$ ). The consistency of this effect over the two samples and its statistical significance for $\mathrm{SX}$ imply that the '+' mutations in A6-9 and B6-27 are partially non-additive in their effects.

\section{Recurrent hybridization and selection}

Following the initial cross (S0) between A6-9 and B6-27, two generations of hybridization were carried out using, in each case, high-titre progeny selected from the previous generation as parents. The highest green-spored and the highest white-spored segregant in S0 were crossed to produce the first selection generation (S1). Forty-five randomly selected S1 
Table 2. Tests for epistasis in crosses $A \times B, A 6-9 \times B 6-27$ and in two subsequent generations of hybridization and selection

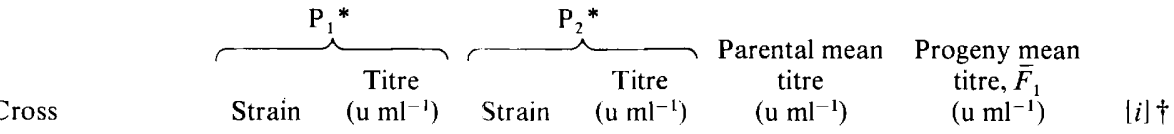

(a) Initial hybridization

$\mathrm{A} \times \mathrm{B}$

A6-9 $\times$ B $6-27$, S0

A6-9 $\times$ B6-27, SX

$\begin{array}{lrlr}\text { A } & 6 \cdot 3 & \text { B } & 5.8 \\ \text { A6-9 } & 11 \cdot 2 & \text { B } 6-27 & 14.4\end{array}$

$14 \cdot 4$

$\begin{array}{llll}\text { A } 6-9 & 18.5 & \text { B } 6-27 & 19.6\end{array}$

$12 \cdot 8$

$19 \cdot 1$

Selection generations

$\mathrm{S} 1$

S0-88

$13 \cdot 9 \quad$ S $0-87 \quad 15 \cdot 1$

$14 \cdot 5$

$16 \cdot 2$

$16 \cdot 3$

* $\mathrm{P}_{1}$, green-spored parent; $\mathrm{P}_{2}$, white-spored parent. $\dagger[i]=\frac{1}{2}\left(\overline{\mathbf{P}}_{1}+\overline{\mathbf{P}}_{2}\right)-\overline{\mathrm{F}}_{1}$.

$\ddagger$ Significantly different from zero: test of significance carried out on $\log \mathrm{u} \mathrm{ml} l^{-1}$.
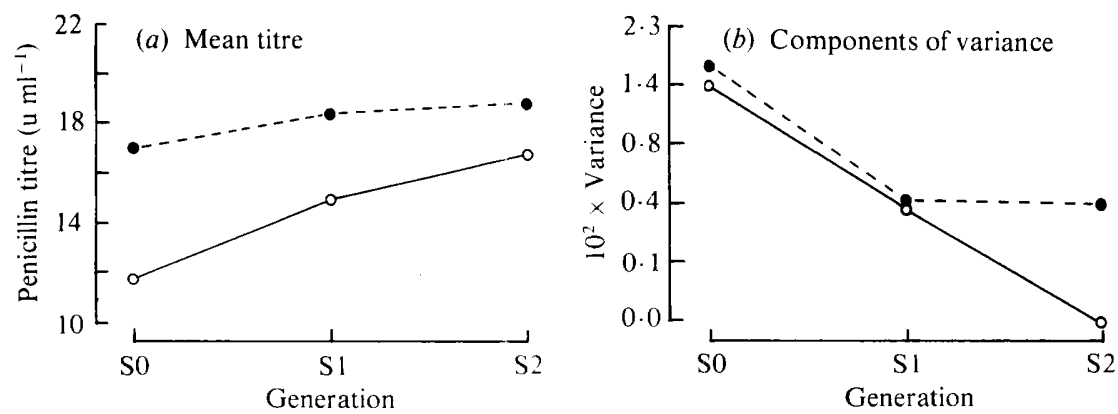

Fig. 2. Response to selection for increased penicillin production following hybridization of A6-9 and B6-27. (a) Changes in the progeny mean titre (O) and in the mean of the highest green- and white-spored segregants, i.e. the strains selected as parents for the next generation $(\boldsymbol{O})$. (b) Changes in the genetic $(O)$ and environmental $(O)$ components of variance.

progeny were each assayed in two replicate shake flasks following the procedure used for SX. On the basis of these results, the highest green-spored and white-spored segregants in S1 were selected and crossed to give the second selection generation (S2). Forty-six S2 progeny were assayed as for the S1 progeny. Selection of parents for the S1 and S2 generations was based on titre and conidial colour without regard to auxotrophic markers. As a result biAl was lost following the S0 generation, but pabaAI continued to segregate in the S1 and S2 generations. Although the requirement for the parents of S1 and S2 to be of different conidial colour prevented selection of the two absolute highest-yielding segregants it avoided the need to reintroduce markers for the identification of hybrid perithecia at each generation. Two replicate flasks of A, B and the appropriate parents were assayed together with the $\mathrm{S} 1$ and $\mathrm{S} 2$ progenies. The titres of $\mathrm{A}$ and $\mathrm{B}$ in $\mathrm{S} 0, \mathrm{~S} 1$ and $\mathrm{S} 2$ were similar and no attempt was made to correct the progeny titres for differences in the fermentation conditions between generations.

The progeny mean titre increased by almost $45 \%$ from $11.8 \mathrm{u} \mathrm{ml}^{-1}$ to $17.0 \mathrm{u} \mathrm{ml}^{-1}$ between S0 and S2 (Fig. 2a). This was accompanied by a fall in the amount of genetic variation in the progenies (Fig. $2 b$ ). Some genetic variation remained after one generation of selection (S1) but none could be detected after two (S2) (Table 3). As in the S0 generation, the markers (pabaAl and $w A 1$ only) had no effect on penicillin titre in the S1 and $\mathrm{S} 2$ generations (Table 3) and the total between strains M.S. has been used to estimate $\sigma_{G}^{2}$. Thus all the genetic variation 
Table 3. Summary of analyses of variation in penicillin titre $\left(\log u \mathrm{ml}^{-1}\right)$ among progenies from two successive generations of hybridization and selection

\begin{tabular}{|c|c|c|c|c|c|c|c|c|c|c|}
\hline \multirow[b]{2}{*}{ Cross } & \multicolumn{2}{|c|}{ Between progeny } & \multicolumn{2}{|c|}{$\begin{array}{c}\text { Between marker } \\
\text { genotypes }\end{array}$} & \multicolumn{2}{|c|}{$\begin{array}{l}\text { Within marker } \\
\text { genotypes }\end{array}$} & \multicolumn{2}{|c|}{ Between blocks } & \multicolumn{2}{|c|}{ Progeny $\times$ blocks } \\
\hline & d.f. & M.S. & d.f. & M.S. & d.f. & M.S. & d.f. & M.S. & d.f. & M.S. \\
\hline S1 & 44 & $0.012^{*}$ & 3 & 0.002 & 41 & $0.012^{*}$ & 1 & $0.028^{*}$ & 44 & 0.004 \\
\hline $\mathrm{S} 2$ & 45 & 0.004 & 3 & 0.001 & 42 & 0.004 & 1 & 0.000 & 45 & 0.004 \\
\hline
\end{tabular}

released by hybridization of the divergent strains had been fixed after two generations; consequently no further response was expected and the programme of recurrent hybridization and selection was terminated. The change from replication of fermentations over experiments (S0) to replication within an experiment (S1 and S2) led to a drop in the environmental component of variation (Fig. $2 b$ ).

The parental mean titres also increased during selection, in this case from $12.8 \mathrm{u} \mathrm{ml}^{-1}$ in $\mathrm{S} 0$ (mean of A6-9 and B6-27) to $16.2 \mathrm{u} \mathrm{ml}^{-1}$ in S2. However, this increase is more apparent than real since the titres of A6-9 and B6-27 in S0 (Table $2 a$ ) were low relative to their average performance $\left(14.2 \mathrm{u} \mathrm{ml}^{-1}\right.$ and $15.5 \mathrm{u} \mathrm{ml}^{-1}$, respectively). The highest yields obtained among the $\mathrm{S} 2$ progeny $\left(18 \mathrm{u} \mathrm{ml}^{-1}\right)$ were higher than the average values for A6-9 and B6-27 but not greater than the maximum titres recorded for these strains. Thus, although the recombination programme gave some improvement over the initial mutation-selected strains, the gain was small, i.e. around $20 \%$ of the mean of A6-9 and B6-27. Estimation of $[i]$ for the $\mathrm{S} 1$ and $\mathrm{S} 2$ generations gave non-significant negative values (Table $2 b$ ) indicating that the genes responsible for the non-additive effects in the SO generation had been fixed after one generation of selection.

\section{DISCUSSION}

Hybridization of A6-9 and B6-27 released considerable genetic variation for penicillin titre indicating that many of the '+' mutations in the two lines involved different genes. In fact the recovery of some progeny with titres equivalent to the ancestral strains $\mathrm{A}$ and $\mathrm{B}$ (Fig. $1 b, c$ ) suggests no overlap between the two sets of mutations. This confirms the conclusion from a complementation test that A6-9 and B6-27 carry non-allelic mutations (Simpson \& Caten, 1980). The involvement of different genes in increasing penicillin titre in independently selected strains of $A$. nidulans has also been found with major mutations (Ditchburn et al., 1976 ) and with natural variation (Merrick, 1975b). Deductions concerning the extent of allelism between divergent strains of $P$. chrysogenum from the results of parasexual crosses are complicated firstly by variation in yield between independent diploids from the same original parents (Macdonald et al., 1965), and secondly by the parental genome segregation observed in these crosses (Macdonald et al., 1964, 1965).

The cross between A6-9 and B6-27 showed strong evidence of non-additive gene action. Some non-additivity of the mutations in these two strains had previously been suggested by the fact that the observed titre of diploid A6-9/B6-27 was lower than that predicted by a model involving additive and dominance effects only (Simpson \& Caten, 1980). Furthermore, backcrosses of A6-9 and B6-27 to the ancestral genotype indicated duplicate interactions within each strain (Simpson \& Caten, 1980). Duplicate epistasis would also account for the A6-9 $\times$ B6-27 progeny distribution, with the difference in the sign of $[i]$ between the two types of cross ( $[i]$ was negative in the backcrosses but positive for A6-9 $\times \mathrm{B} 6-27)$ resulting from the fact that the parents for the backcrosses were in coupling whilst those for A6-9 $\times$ B6-27 were in repulsion. (The values of $[i]$ for the backcrosses given by Simpson \& Caten, 1980, are in $\log \mathrm{u} \mathrm{ml}^{-1}$.) The results for the backcrosses and A6-9 $\times$ B6-27 therefore suggest that on 
average the individual ' + ' mutations selected during the mutagenesis programme exert their biggest effects on a low-titre background and that accumulation of mutations through mutagenesis or recombination produces progressively decreasing gains. However, this epistatic component cannot account for all the genetic variation since segregants with higher titres than the parents were recovered from A6-9 $\times$ B6-27, indicating an additive component. Indeed, some additivity is essential for the selection of successive mutations in a recurrent mutagenesis programme. Estimation of the relative contribution of these additive and non-additive effects to the genetic variation would require raising further generations (Mather \& Jinks, 1971).

Three major mutations increasing penicillin yield in $A$. nidulans also interacted in a duplicate manner such that recombinant strains carrying pairwise combinations of these mutations produced no more penicillin than strains carrying the higher-yielding single mutation on its own (Ditchburn et al., 1976). In addition, results similar to those for A6-9 $\times$ B6-27 were obtained from a cross between two divergent lines of Cephalosporium acremonium, with many progeny producing less cephalosporin $\mathrm{C}$ than the lower-yielding parent but few producing more than the higher-yielding parent (Hamlyn \& Ball, 1979). However, one segregant among 600 tested gave a $40 \%$ yield improvement over the higher-yielding parent, demonstrating that valuable gains could still be achieved through recombination. Not all induced mutations increasing antibiotic yield show such undesirable interactions. Ball (1973) reported that parasexual segregants of $P$. chrysogenum carrying two independently induced mutations $\left(t_{2}\right.$ and $\left.t_{5}\right)$ gave penicillin titres in excess of any recorded previously. Hence the effects of $t_{2}$ and $t_{5}$ appear additive or even complementary.

Two generations of selection from the progeny of A6-9 $\times$ B6-27 gave only a small further increase in productivity. Small realized gains following hybridization of divergent lines may result from several factors including: (1) overlap of mutations, (2) low heritabilities, (3) failure of recombination, (4) epistasis and (5) genetic drift (see Soller, 1980, for elaboration of 1, 4 and 5). Let us consider the possible impact of each of these factors on this recombination and selection programme. It has been concluded above that the ' + ' mutations in A6-9 and B6-27 involve different genes. Estimates of heritability (Merrick \& Caten, 1975) for the A6-9 $\times$ B6-27 crosses (S0, $\left.h^{2}=0.45 ; \mathrm{SX}, h^{2}=0.55\right)$ are similar to those for populations produced by crossing wild-type isolates (Merrick \& Caten, 1975; Merrick, 1975 a), by crossing selected lines (Merrick, 1975 b) and by mutagen treatment (Simpson \& Caten, 1979a); selection was highly effective from these latter populations. Failure of recombination may arise through inefficiency of the genetic system employed or linkage. Meiotic recombination in $A$. nidulans is highly efficient (Cove, 1977) and the markers in A6-9 $\times$ B6-27 recombined as expected. Furthermore, the recovery of low-titre segregants (Fig. $1 b, c$ ) in small progeny samples indicates that the titre alleles are not linked. Factors 1,2 and 3 did not therefore apply. As regards factor 4 , however, there is strong evidence that the ' + ' mutations interact in a duplicate manner. These interactions would markedly reduce the yield advance achieved by selecting segregants carrying all or most ' + ' mutations relative to that expected with an additive system. Since only two parents were selected in each generation it is likely that some favourable alleles were lost by chance (factor 5). These considerations suggest that the small magnitude of the gains from hybridization of A6-9 and B6-27 was due primarily to epistasis, with genetic drift as a possible secondary factor. This conclusion is supported by comparisons with a selection programme carried out using natural genetic variation for penicillin production in $A$. nidulans (Merrick, $1975 a, b$ ). In this case hybridization of independently selected lines allowed a further substantial increase in titre. The methods used by Merrick were virtually identical to those used here and both his and the present inter-line cross indicated little overlap of mutations and similar heritabilities. The major difference was in the type of gene action which was predominantly additive for the natural variation. This contrast between the genetic architecture of induced and natural polygenic systems suggests that the latter is a selected sample of the allelic differences that can be generated by mutagenesis. 
The selection of A6-9 and B6-27 (Simpson \& Caten, 1979 b) and the subsequent selection amongst their progeny described here may be considered as a single strain development programme with a phase of recurrent mutagenesis and a phase of recurrent recombination, each consisting of several cycles. The largest gains per cycle were realized during the mutagenesis phase and, as a means of generating the most productive strain with the resources available, the division of the mutagenesis phase into two parallel lines and the subsequent hybridization of these lines was not effective. For the same total effort a single mutation line could have been carried through 15 cycles and would have undoubtedly shown substantially greater improvement. This contrasts with the conclusion of Soller (1980) that where the genetic variation due to mutagenesis $\left(\sigma_{M}^{2}\right)$ is large relative to the measurement errors $\left(\sigma_{E}^{2}\right)$ (i.e. $\left.\sigma_{M}^{2} \geqslant \sigma_{E}^{2}\right)$ and the degree of overlap of the mutations is small, both conditions which apply in the present case, a combined programme of mutagenesis and recombination should show a greater gain than a programme based on mutagenesis alone. However, Soller (1980) did recognize that the advantage of the combined programme would be less in the presence of epistasis and in the absence of a deleterious effect of mutagenesis on the population mean. Mutagenesis did depress the mean titre of the $A$. nidulans populations but this reduction was small and probably due to the average greater effect of titre-decreasing mutations rather than to their higher frequency (Simpson \& Caten, 1979 $a$ ).

These genetic analyses of A6-9 and B6-27 point to the development under recurrent mutagenesis of complex polygenic systems and suggest that unfavourable gene interactions may limit the gains that can be achieved through recombination of divergent lines. Whether similar genetic control would evolve with other organisms and products, or indeed with different mutagenic treatments, can only be determined by experiment. Further quantitative genetic studies of antibiotic yield are required to establish general values for the relevant parameters and to aid choice of the best combination of mutation and recombination in strain development.

We thank Glaxo-Group Research Ltd, Sefton Park, Stoke Poges, Buckinghamshire for the provision of materials and the Glaxo staff, especially Dr C. Ball, Dr J. P. R. Herrmann and Mr R. Crosse, for their guidance and encouragement. One of us (I. N.S.) gratefully acknowledges a Science Research Council CASE studentship.

\section{REFERENCES}

Alikhanian, S. I. (1970). Applied aspects of microbial genetics. Current Topics in Microbiology and Immunology 53, 91-148.

BALL, C. (1971). Haploidization analysis in Penicillium chrysogenum. Journal of General Microbiology 66, 63-69.

BALl, C. (1973). The genetics of Penicillium chrysogenum. Progress in Industrial Microbiology 12, 47-72.

BALL, C. (1978). Genetics in the development of the penicillin process. In Antibiotics and Other Secondary Metabolites, pp. 165-176. Edited by R. Hütter, T. Leisinger, J. Núesch \& W. Wehrli. London: Academic Press.

Calam, C. T. (1964). The selection, improvement and preservation of micro-organisms. Progress in Industrial Microbiology 5, 1-53.

Caten, C. E. (1979). Quantitative genetic variation in fungi. In Quantitative Genetic Variation, pp. 35-59. Edited by J. N. Thompson, Jr \& J. M. Thoday. New York: Academic Press.

Caten, C. E. \& Jinks, J. L. (1976). Quantitative genetics. In Second International Symposium on the Genetics of Industrial Microorganisms, pp. 93-111.
Edited by K. D. Macdonald. London: Academic Press.

Clutterbuck, A. J. (1973). Gene symbols in Aspergillus nidulans. Genetical Research 21, 291296.

Cove, D. J. (1977). The genetics of Aspergillus nidulans. In Genetics and Physiology of Aspergillus, pp. 81-95. Edited by J. E. Smith \& J. A. Pateman. London: Academic Press.

DAvies, O. L. (1964). Screening for improved mutants in antibiotic research. Biometrics 20, 576-591.

DEMAIN, A. L. (1973). Mutation and the production of secondary metabolites. Advances in Applied Microbiology 16, 177-202.

Ditchburn, P., Holt, G. \& Macdonald, K. D. (1976). The genetic location of mutations increasing penicillin yield in Aspergillus nidulans. In Second International Symposium on the Genetics of Industrial Microorganisms, pp. 213-227. Edited by K. D. Macdonald. London: Academic Press.

Elander, R. P. \& Espenshade, M. A. (1976). The role of microbial genetics in industrial microbiology In Industrial Microbiology, pp. 192-256. Edited by B. M. Miller \& W. Litsky. New York: McGraw-Hill. 
Elander, R. P., Espenshade, M. A., Pathak, S. G. \& PAN, C. H. (1973). The use of parasexual genetics in an industrial strain selection programme with Penicillium chrysogenum. In Genetics of Industrial Microorganisms, vol. II, pp. 239-253. Edited by Z. Vanek, Z. Hostálek \& J. Cudlin. Prague: Academia.

Elander, R. P., Chang, L. T. \& Vaughan, R. W. (1977). Genetics of industrial microorganisms. Annual Reports on Fermentation Processes 1, 1-40.

HAMlyN, P. F. \& BALL, C. (1979). Recombination studies with Cephalosporium acremonium. In Genetics of Industrial Microorganisms, pp. 185191. Edited by O. K. Sebek \& A. I. Laskin. Washington, D.C.: American Society for Microbiology.

Hopwood, D. A. (1977). Genetic recombination and strain improvement. Developments in Industrial Microbiology 18, 9-21.

Hopwood, D. A. \& ChATER, K. F. (1980). Fresh approaches to antibiotic production. Philosophical Transactions of the Royal Society, Series B 290 , 313-328.

Hopwood, D. A. \& Merrick, M. J. (1977). Genetics of antibiotic production. Bacteriological Reviews $\mathbf{4 1}$, $595-635$.

MacDonald, K. D. (1966). Differences in diploids synthesized between the same parental strains of Penicillium chrysogenum. Antonie van Leeuwenhoek 32, 431-441.

Macdonald, K. D. \& Holt, G. (1976). Genetics of biosynthesis and overproduction of penicillin. Science Progress 63, 547-573.

Macdonald, K. D., Hutchinson, J. M. \& Gillett, W. A. (1964). Properties of heterozygous diploids between strains of Penicillium chrysogenum selected for high penicillin yield. Antonie van Leeuwenhoek 30, 209-224.

Macdonald, K. D., Hutchinson, J. M. \& Gillett,
W. A. (1965). Heterozygous diploids of Penicillium chrysogenum and their segregation patterns. Genetica 36, 378-397.

MATHER, K. \& JINKS, J. L. (1971). Biometrical Genetics, 2nd edn. London: Chapman and Hall.

MerricK, M. J. (1975a). Hybridization and selection for increased penicillin titre in wild-type isolates of Aspergillus nidulans. Journal of General Microbiology 91, 278-286.

MERRICK, M. J. (1975 b). The inheritance of penicillin titre in crosses between lines of Aspergillus nidulans selected for increased productivity. Journal of General Microbiology 91, 287-294.

Merrick, M. J. \& CATEN, C. E. (1975). The inheritance of penicillin titre in wild-type isolates of Aspergillus nidulans. Journal of General Microbiology 86, 283-293.

SERMONTI, G. (1959). Genetics of penicillin production. Annals of the New York Academy of Sciences 81, 950-966.

SERMONTI, G. (1969). Genetics of Antibiotic-producing Microorganisms. London: Wiley-Interscience.

Simpson, I. N. \& CATEN, C. E. (1979a). Induced quantitative variation for penicillin titre in clonal populations of Aspergillus nidulans. Journal of General Microbiology 110, 1-12.

Simpson, I. N. \& Caten, C. E. (1979b). Recurrent mutation and selection for increased penicillin titre in Aspergillus nidulans. Journal of General Microbiology 113, 209-217.

Simpson, I. N. \& CATEN, C. E. (1980). Genetics of penicillin titre in lines of Aspergillus nidulans selected through recurrent mutagenesis. Journal of General Microbiology 121, 5-16.

SOLLER, M. (1980). Optimisation of recurrent selection under mutagenesis in micro-organisms. Heredity 44, 295-307. 